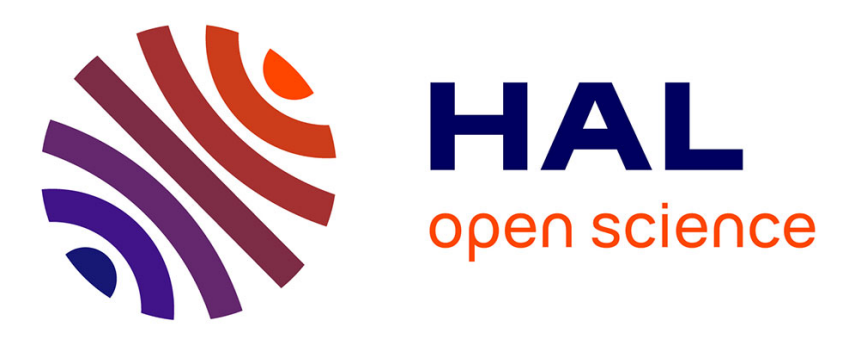

\title{
Estimation d'abondance des criquets (Orthoptera: Acrididae) dans les écosystèmes prairiaux
}

\author{
Isabelle Badenhausser
}

\section{To cite this version:}

Isabelle Badenhausser. Estimation d'abondance des criquets (Orthoptera: Acrididae) dans les écosystèmes prairiaux. Annales de la Société Entomologique de France, 2012, 48 (3-4), pp.397-406. hal01137006

\section{HAL Id: hal-01137006 \\ https://hal.science/hal-01137006}

Submitted on 30 Mar 2015

HAL is a multi-disciplinary open access archive for the deposit and dissemination of scientific research documents, whether they are published or not. The documents may come from teaching and research institutions in France or abroad, or from public or private research centers.
L'archive ouverte pluridisciplinaire HAL, est destinée au dépôt et à la diffusion de documents scientifiques de niveau recherche, publiés ou non, émanant des établissements d'enseignement et de recherche français ou étrangers, des laboratoires publics ou privés. 


\title{
Estimation d'abondance des criquets (Orthoptera : Acrididae) dans les écosystèmes prairiaux
}

ISABELLE BADENHAUSSER

CEBC - CNRS (UPR 1934), Villiers en Bois, F-79360, Beauvoir sur Niort, France INRA, USC1339, CEBC, Villiers en Bois, F-79360, Beauvoir sur Niort, France

\begin{abstract}
Estimation of grashopper abundance in grassland ecosystems (Orthoptera: Acrididae). Grasshoppers form an important component of the trophic chain and are considered as good indicators of agricultural change and its environmental consequences. They are among the major inhabitants of grasslands and are thought to decline in intensive agricultural landscape due to habitat fragmentation and loss, as well as agricultural practices. Estimates of acridid population parameters are essential in ecological studies and in management strategies, especially for implementation of conservation schemes. In this paper we describe a sampling technique and a sampling plan to estimate grasshopper densities in grassland ecosystems. We used a 7-yr data set consisting in grasshopper surveys in 916 grasslands in a $450-\mathrm{km}^{2}$ study site located in an intensive agricultural landscape. We establish that a $1 \mathrm{~m}^{2}$ cage sampler provides robust estimates of grasshopper densities in regard to temperature during sampling operations. Moreover, we provide sampling plans based on count data to estimated grasshopper densities per $\mathrm{m}^{2}$. A random sampling plan can be used to estimate grasshopper densities based on variance-mean relationships. Relative precisions that could be reached, when using sample size of 15 replicates, range between $50 \%$ when acridid densities are lower than $1 / \mathrm{m}^{2}$ and $20 \%$ when they are higher than $7 / \mathrm{m}^{2}$. The importance of taking into account population dynamics in the sampling process is discussed.
\end{abstract}

Résumé. Les criquets représentent un taxon central dans les chaînes alimentaires et sont de bons indicateurs à la fois des caractéristiques des milieux et des perturbations de leurs habitats. Associés exclusivement aux habitats herbacés pérennes, ils sont menacés dans les zones d'agriculture intensive. Du fait de leur importance dans les réseaux trophiques, notamment comme ressources alimentaires pour un grand nombre d'espèces aviaires, ils font l'objet d'une attention croissante des écologistes et des gestionnaires des milieux dans le cadre d'études de conservation pour évaluer quantitativement leurs populations. L'objectif de cette étude est de décrire une technique d'échantillonnage et un plan d'échantillonnage destinés à estimer la densité de criquets dans les milieux prairiaux. Cette étude se base sur 7 années d'observations de terrain, menées sur un vaste site d'étude en plaine agricole intensive. Nous montrons que le biocénomètre d'1 $\mathrm{m}^{2}$ de base est une technique robuste vis-à-vis des conditions de température durant l'échantillonnage. L'étude établit pour l'ensemble des espèces et pour deux espèces particulières, Pezotettix giornae et Calliptamus italicus, la relation qui lie la variance et la moyenne des effectifs par $\mathrm{m}^{2}$. C'est grâce à cette relation qu'on peut établir les tailles d'échantillons qui permettent d'atteindre des objectifs de précision choisis pour les estimateurs de la densité. Nous montrons que la réalisation de 15 lancers aléatoirement par parcelle permet d'obtenir des estimations de la densité de criquets $/ \mathrm{m}^{2}$ dont la précision, définie par l'intervalle de confiance, varie selon la densité de $50 \%$ pour les densités inférieures à $1 / \mathrm{m}^{2}$ à $30 \%$ pour les densités de 2 à $7 / \mathrm{m}^{2}$ et à $20 \%$ pour les densités supérieures à $7 / \mathrm{m}^{2}$.

Keywords: Sampling, Gomphocerinae, Calliptamus italicus, Pezotettix giornae.

$\mathrm{L}$ es effets négatifs de l'intensification agricole sur la biodiversité européenne (Green et al. 2005) ou nationale (Inchausti \& Bretagnolle 2005) ne sont plus discutés, qu'il s'agisse de plantes, insectes, oiseaux ou de mammifères. Dans les agroécosystèmes, les cultures à végétations pluriannuelles se distinguent radicalement des cultures annuelles sur les plans du niveau et de la fréquence des perturbations qu'elles subissent.

E-mail: badenh@cebc.cnrs.fr

Accepté le 30 août 2012
Malgré la faible surface qui leur est consacrée dans les agrosystèmes intensifs, ces milieux sont essentiels car ils permettent à de nombreux organismes de réaliser leur cycle biologique. Les Orthoptères, en particulier les criquets (Orthoptera: Acrididae), forment une composante importante des écosystèmes prairiaux. Ce sont des proies de prédilection pour de nombreux prédateurs invertébrés (araignées, autres insectes) et vertébrés (reptiles, mammifères, oiseaux), en particulier pour les oiseaux (Barker 2004) dont le déclin est pour partie expliqué par la raréfaction de la ressource alimentaire (Barker 2004; Bretagnolle et al. 2011). Du 
fait de leur herbivorie, les criquets sont très dépendants de la végétation, ce qui en fait des indicateurs de plus en plus reconnus pour traduire, de par la structure de leurs communautés, le niveau de perturbation anthropique et la qualité des milieux (Wingerden et al. 1992; Baldi \& Kisbenedek 1997; Gardiner et al. 2005).

Quantifier l'abondance des criquets est un objectif poursuivi pour la gestion des populations, notamment dans le cadre d'études à but conservatoire, cellesci étant les plus fréquentes en France du fait du faible potentiel de dégâts aux cultures des espèces qui y sont rencontrées. De nombreuses méthodes d'échantillonnage ont été décrites pour déterminer l'abondance des criquets (Orthoptera: Acrididae) dans des gammes variées de milieux prairiaux (Onsager 1977, Gardiner et al. 2005, Schirmel et al. 2010, Doxon et al. 2011). Il s'agit par ordre décroissant de fréquence d'utilisation, du filet fauchoir, du quadrat ouvert (Gardiner et al. 2002), de la notation visuelle le long de transects (Voisin 1986), et de la cage de nuit qui est mentionnée comme étant la référence en milieu herbacé (Onsager 1977 ; Evans et al. 1983 ; Browde et al. 1992) mais elle est très couteuse en temps. La cage de jour (Onsager 1977 ; Gardiner et al. 2005), dénommée biocénomètre, est une enceinte ouverte en haut et en bas et de hauteur suffisante pour permettre la capture des criquets en évitant leur fuite. Cette méthode donne des estimations très proches de celles données par la cage de nuit (Onsager 1977) et apparaît comme une excellente alternative. Elle s'applique à des espèces non rares et à pouvoir de déplacement limité, et peut être employée dans d'autres habitats que les prairies comme l'a montré Guegen et al. (1980) dans différents types de landes. Sa surface, sa mise en œuvre ainsi que le protocole d'échantillonnage ne font pas l'objet d'un consensus, ce qui explique que cette technique reste encore peu utilisée (5\% des études publiées utilisent cette méthode selon Gardiner et al. 2005).

La majorité des études sur les méthodes d'échantillonnage des criquets mentionnent des résultats fiables et reproductibles mais dans des conditions d'utilisation restrictives, liées essentiellement à la densité de criquets, à la structure de la végétation et aux conditions climatiques (Richards \& Waloff 1954). Il apparaît donc important de développer une technique standardisée autorisant des comparaisons entre études, pouvant être mise en œuvre par un grand nombre d'utilisateurs, dans des conditions variées notamment de densités de criquets et de structures de végétation, et dans une gamme assez large de conditions météorologiques (Gardiner et al. 2005).

Le but de notre étude est de diffuser des résultats issus de notre expérience de terrain pour l'estimation d'abondance des criquets des milieux prairiaux, en se fondant sur la technique du biocénomètre d' $1 \mathrm{~m}^{2}$. En effet, dans une étude précédente (Badenhausser $e t$ al. 2007) nous avons montré qu'un biocénomètre de taille inférieure sous-estime les densités de criquets par rapport à un biocénomètre d' $1 \mathrm{~m}^{2}$. Nous proposons i/ de fournir une description permettant la réalisation d'un biocénomètre démontable; ii/ d'établir sa robustesse par rapport à la température durant l'échantillonnage; iii/ de proposer un protocole d'échantillonnage définissant la précision associée à l'estimation de la densité de criquets $/ \mathrm{m}^{2}$ en fonction du nombre de lancers réalisés par parcelle. Enfin, nous discutons de l'importance de la date d'échantillonnage en rapport avec la dynamique des populations et les objectifs poursuivis. Notre étude se fonde sur un échantillonnage pluri-annuel (de 2004 à 2010) réalisé à grande échelle, dans un paysage agricole de plaine céréalière intensive où dominent la sous famille des Gomphocerinae, l'espèce Pezotettix giornae (Rossi) (Catantopinae) et l'espèce C. italicus (L.) (Calliptaminae) (Badenhausser et al. 2009).

\section{Matériel et Méthodes}

\section{La zone d'étude}

Les données ont été collectées dans la Zone Atelier «Plaine et Val de Sèvre ", plaine céréalière intensive de $450 \mathrm{~km}^{2}$ localisée dans la région Poitou-Charentes, au sud de Niort, près de Chizé (Ouest de la France, $46^{\circ} 07^{\circ} \mathrm{N} 0^{\circ} 17^{\circ} \mathrm{W}$ ). Cette zone comprend 19000 parcelles agricoles et se situe dans un paysage dominé par les céréales, le colza, le maïs et le tournesol. Les prairies y représentent $12 \%$ de la surface et sont des prairies semées artificielles, essentiellement de la luzerne, ou de Poacées pures ou en association. Leur durée d'implantation est relativement faible (3 à 4 ans en moyenne), et elles sont exploitées par la fauche ou la pâture. Cette zone est fortement instrumentalisée depuis 1994, et toutes les parcelles font l'objet d'un suivi annuel de leur mode d'occupation du sol. De plus, la zone est le support de suivis à long terme d'un grand nombre de taxons depuis les oiseaux jusqu'à leurs ressources alimentaires, plantes, micromammiferes et insectes. L'ensemble des données collectées est géo-référencé dans une base ArcGis (ESRI 2006).

\section{Les espèces de criquets de la zone d'étude}

Depuis 2004, l'ensemble des suivis que nous avons réalisés sur la Zone Atelier a permis de détecter environ 30 espèces de criquets (les Tetrigidae n'ont pas été déterminées à l'espèce) à des densités extrêmement variables selon les années et les espèces. La sousfamille des Gomphocerinae y est la plus représentée avec près de $80 \%$ des effectifs quelle que soit l'année. Les espèces les plus couramment observées sont par ordre décroissant d'abondance et toutes années confondues, Euchorthippus elegantulus Zeuner, Chorthippus biguttulus (L.), Pezottetix giornae, Chorthippus parallelus (Zetterstedt), Chorthippus albomarginatus (De Geer), Chorthippus dorsatus (Zetterstedt) ainsi que Calliptamus italicus. Des espèces moins abondantes, mais néanmoins fréquentes sont représentées par Omocestus rufipes (Zetterstedt), Euchorthippus declivus (Brisout de Barneville) et Chorthippus brunneus 
Tableau 1. Température durant l'échantillonnage (moyenne et Erreur Standard) et densités de criquets $/ \mathrm{m}^{2}$ (moyenne et Erreur Standard) de la sous-famille des Gomphocerinae, de Pezotettix giornae, Calliptamus italicus et densités totales (moyenne et Erreur Standard) (Nombre de parcelles échantillonnées $=n$ ).

\begin{tabular}{cccccc}
\hline Année $(\boldsymbol{n})$ & $\begin{array}{c}\text { Température } \\
(\mathrm{m} \pm \mathrm{SE})\end{array}$ & $\begin{array}{c}\text { Gomphocerinae } \\
(\mathrm{m} \pm \mathrm{SE})\end{array}$ & $\begin{array}{c}\text { Pezotettix giornae } \\
(\mathrm{m} \pm \mathrm{SE})\end{array}$ & $\begin{array}{c}\text { Calliptamus italicus } \\
(\mathrm{m} \pm \mathrm{SE})\end{array}$ & $\begin{array}{c}\text { Total criquets } \\
(\mathrm{m} \pm \mathrm{SE})\end{array}$ \\
\hline $\mathbf{2 0 0 4}(\mathbf{4 5})$ & $26,5 \pm 0,8$ & $4,24 \pm 0,82$ & $0,42 \pm 0,11$ & $0,37 \pm 0,12$ & $5,13 \pm 0,84$ \\
$\mathbf{2 0 0 5}(\mathbf{1 0 1})$ & $26,7 \pm 0,4$ & $3,37 \pm 0,46$ & $0,36 \pm 0,07$ & $0,26 \pm 0,09$ & $4,11 \pm 0,50$ \\
$\mathbf{2 0 0 6}(\mathbf{9 9})$ & $27,7 \pm 0,5$ & $3,93 \pm 0,72$ & $0,55 \pm 0,13$ & $0,50 \pm 0,08$ & $5,00 \pm 0,78$ \\
$\mathbf{2 0 0 7}(\mathbf{1 7 9})$ & $23,5 \pm 0,2$ & $1,32 \pm 0,16$ & $0,46 \pm 0,07$ & $0,11 \pm 0,04$ & $1,96 \pm 0,22$ \\
$\mathbf{2 0 0 8}(\mathbf{2 2 1})$ & $24,1 \pm 0,2$ & $0,62 \pm 0,07$ & $0,12 \pm 0,03$ & $0,02 \pm 0,01$ & $0,77 \pm 0,09$ \\
$\mathbf{2 0 0 9}(\mathbf{1 3 0})$ & $24,6 \pm 0,3$ & $1,01 \pm 0,17$ & $0,18 \pm 0,04$ & $0,05 \pm 0,02$ & $1,27 \pm 0,19$ \\
$\mathbf{2 0 1 0}(\mathbf{1 4 1 )}$ & $24,3 \pm 0,4$ & $2,52 \pm 0,40$ & $0,29 \pm 0,06$ & $0,14 \pm 0,05$ & $2,97 \pm 0,42$ \\
\hline
\end{tabular}

(Thunberg). Enfin, certaines espèces apparaissent rarement ou sont associées à des milieux humides ou arides. Il s'agissait pour les premiers d'Aiolopus strepens (Latreille), Mecostethus parapleurus (Hagenbach), Stethophyma grossum (L.), et Paracinema tricolor (Thunberg) et pour les seconds d'Oedipoda caerulescens (L.), Aiolopus thalassinus (F) et Sphingonotus caerulans (L). Au moins trois espèces de Tetrigidae ont été rencontrées. Toutes ces espèces sont monovoltines à l'exception d'O. rufipes et pondent leurs œufs sous forme d'oothèques dans le sol ou à la base des plantes.

\section{Les parcelles d'étude}

Les données proviennent de sept années d'échantillonnage, de 2004 à 2010. Chaque année (à part en 2004), un échantillon d'un minimum de 100 parcelles de prairies a été tiré au hasard au sein de la Zone Atelier parmi les prairies artificielles (majoritairement de la luzerne), les prairies de Poacées pures ou en association et les jachères spontanées (Tab. 1). Ce protocole de sélection des parcelles permet d'avoir une bonne représentativité de la densité annuelle de criquets des prairies de la zone d'étude. Au total 916 parcelles ont été échantillonnées.

\section{L'échantillonnage et les notations}

Chaque parcelle a fait l'objet de l'échantillonnage des populations de criquets à une date correspondant chaque année au maximum des populations d'adultes. Cette date, déterminée grâce à des parcelles faisant l'objet d'un suivi hebdomadaire, se situait entre la dernière semaine de juillet et le 15 août selon les années. Les échantillonnages étaient réalisés sur une période maximale de 10 à 15 jours, permettant de ne pas avoir trop de variations liées à la dynamique des populations (Badenhausser et al. 2009). Un échantillonnage était effectué avec la technique du biocénomètre d' $1 \mathrm{~m}^{2}$ lancé 10 fois au hasard par parcelle. A chaquelancer, les individus capturésàl'intérieur dubiocénomètre avec un petit filet de pêche étaient dénombrés visuellement sur le terrain et classés en quatre groupes, tout en distinguant les juvéniles des adultes: la sous-famille des Gomphocerinae, $P$. giornae, C. italicus et toutes les autres espèces. Un individu sur 5 ou sur 10 était prélevé pour identification ultérieure. Compte tenu du nombre élevé de parcelles sélectionnées, et du fait que la période d'échantillonnage devait être la plus courte possible, plusieurs équipes de deux personnes réalisent en parallèle les échantillonnages. Une étude antérieure a montré que ce facteur pouvait être négligé (Badenhausser et al. 2009). La température au moment de l'échantillonnage a été notée sauf en 2004.

\section{Description du biocénomètre}

La cage employée possède une base carrée d' $1 \mathrm{~m}$ de côté et une hauteur de $70 \mathrm{~cm}$. Elle est composée de deux cadres carrés d'1 $\mathrm{m}$ de côté (Fig. 1). Chaque cadre est réalisé en cintrant à chaud un tube de fer creux de $4 \mathrm{~m}$ de long et de $12 \mathrm{~mm}$ de diamètre extérieur, qui est soudé pour fermer le carré. Un petit morceau de tube de même qualité mais de section plus petite $(10 \mathrm{~mm}$ de diamètre extérieur) et de $7 \mathrm{~cm}$ de hauteur est soudé aux quatre angles de chaque cadre. Ces petits tubes sont destinés à recevoir les montants de la cage. Les montants, au nombre de quatre, sont en tube de fer de $70 \mathrm{~cm}$ de haut et de $12 \mathrm{~mm}$ de diamètre. La housse qui ferme latéralement la cage est confectionnée avec un morceau de brise-vent de $4 \mathrm{~m}$ de large. On prévoit, en plus de la hauteur de $70 \mathrm{~cm}$, une bande de $30 \mathrm{~cm}$ qui, entaillée aux 4 angles, forme 4 revers qui permettent au montage de la cage de recevoir et de maintenir en place le cadre supérieur. On coud sur toute la longueur du bord inférieur de la housse une bande de toile bâchée de $20 \mathrm{~cm}$ de largeur qui sert à rendre hermétique la cage posée au sol. Un morceau de bois est glissé à l'extrémité de chaque revers dans un rebord cousu. Un morceau de ficelle de $20 \mathrm{~cm}$ environ est plié en deux, noué et attaché à chaque extrémité des morceaux de bois. La housse est maintenue sur le cadre inférieur grâce à des liens plastiques tous les $10 \mathrm{~cm}$. Un tendeur bien dur de 0,90 m de long, divisé en deux parties égales,

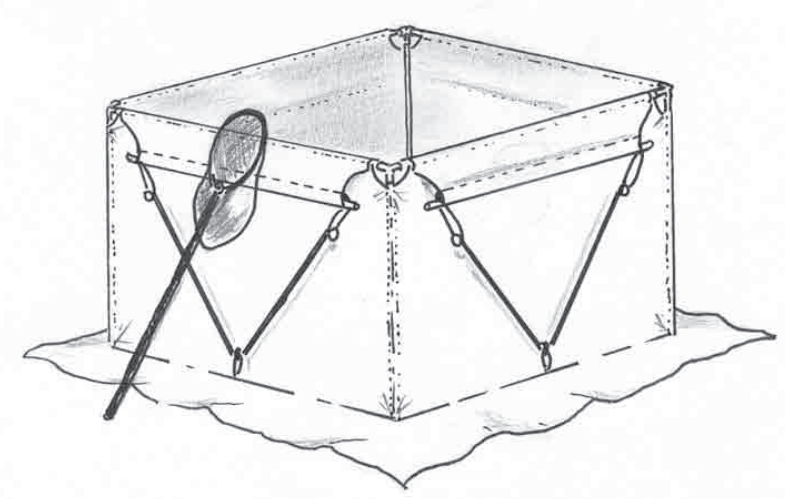

Figure 1

Schéma montrant un modèle de biocénomètre d' $1 \mathrm{~m}^{2}$ démontable en deux cadres carrés d'1 $\mathrm{m}$ de côté et en quatre montants de $70 \mathrm{~cm}$ de hauteur (Réalisation du dessin : Julien Amouret). 
chacune se terminant par un gros nœud, est attaché en un point au milieu de chaque face sur le cadre inférieur et la housse grâce à un lien en plastique. Au montage, chaque nœud est passé dans une des deux ficelles accrochées aux extrémités du morceau de bois du revers. Bien tendu, l'ensemble est rigidifié et démontable rapidement en 2 cadres (dont l'un porte la housse) et 4 tubes. On peut rajouter à cet ensemble un cadre léger d'1 $\mathrm{m}$ de côté en grillage plastique fin, non opaque, qu'on peut encastrer sur le dessus de la cage avant de la lancer. Ce cadre évite la fuite des individus au moment du lancer. Il doit être rigide pour pouvoir le retirer sans emprisonner de criquets dans les plis du tissu.
Effet de la température d'échantillonnage sur l'estimation d'abondance des criquets

Aucune contrainte n'a été mise sur l'heure d'échantillonnage comprise entre 8 heures et 20 heures, ni sur la température requise pour l'échantillonnage. Les relevés n'ont pas eu lieu sous la pluie ou juste après la pluie. Nous posons l'hypothèse que les estimations de densités de criquets obtenues par parcelle échantillonnée ne dépendent pas de la température au moment de l'échantillonnage. Pour la tester, nous avons utilisé indépendamment chaque année le modèle linéaire suivant après
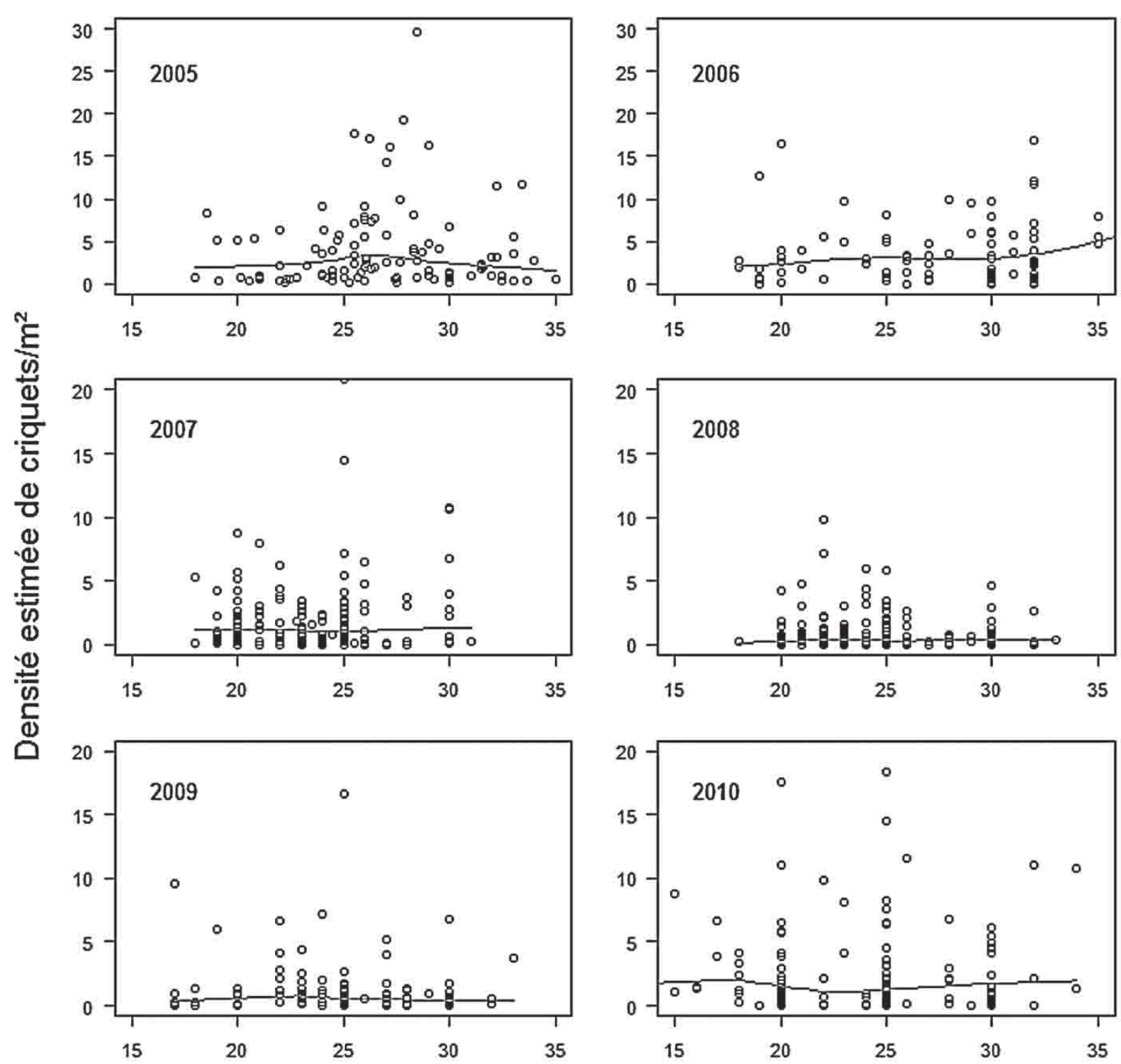

Température durant l'échantillonnage

Figure 2

Représentation de la densité de criquets $/ \mathrm{m}^{2}$ (toutes espèces confondues) estimée dans les parcelles échantillonnées en fonction de la température au moment de l'échantillonnage et de la courbe de tendance intra-annuelle. 
avoir vérifié graphiquement que les effets de la température et de la date d'échantillonnage étaient linéaires :

$$
D=a^{\prime}+b^{\prime} t+c^{\prime} G L
$$

Où $D$ est la densité de criquets $/ \mathrm{m}^{2}$ estimée pour chaque parcelle d'une année donnée en faisant la moyenne des comptages des 10 lancers par parcelle et transformée ensuite en utilisant la transformation en double racine carrée (Dangerfield et al. 2003), $\theta$ est la variable continue de la température au moment de l'échantillonnage (Tab. 1), GL est un facteur à deux niveaux qui distingue les prairies de Poacées des prairies de luzerne, $t$ est le numéro d'ordre du jour de l'échantillonnage dans l'année (= date julienne). L'analyse a porté sur les 796 des 916 parcelles pour lesquelles on disposait de la température durant l'échantillonnage (l'année 2004, aucun relevé de température n'a été fait). Les données ont été analysées avec le logiciel $\mathrm{R}$, version 2.13.00 (R Development Core team 2011).

\section{Plan d'échantillonnage}

La précision associée à l'estimation de la densité de criquets $/ \mathrm{m}^{2}$ de prairie dépend de la variabilité des comptages entre lancers. C'est sur la base d'une estimation de la variabilité entre unités d'échantillonnage que se construit un plan d'échantillonnage, c'est à dire la procédure de sélection des unités d'échantillonnage et leur nombre. Le premier point repose sur une analyse de la structuration spatiale des comptages à l'échelle des parcelles. En effet, de nombreux auteurs ont montré que l'utilisation de l'habitat ne se fait pas de manière aléatoire car il est hétérogène et fournit des micro-habitats qui sont plus ou moins favorables aux différentes espèces aux différents moments de leurs cycles biologiques (Guido \& Gianelle 2001). Toutefois, celle-ci n'est pas prévisible sur une parcelle inconnue et ne peut donc pas être prise en compte pour structurer l'échantillonnage. De ce fait, nous avons choisi de développer une procédure d'échantillonnage aléatoire qui, bien que moins puissante que d'autres procédures, fournit des estimations non biaisées en toutes circonstances. Le second point, le nombre de lancers à réaliser, repose sur la connaissance de la variabilité des comptages entre lancers. Des études antérieures ont montré que celle-ci variait en fonction de la densité de la population (Badenhausser et al. 2007), la variance entre comptages augmentant avec la densité selon une relation exponentielle, la relation de Taylor (1961) $S^{2}=a D^{b}$, que l'on peut linéariser pour en calculer les paramètres:

$$
\text { Eq } 1: \log _{10} S^{2}=\log _{10} a+b \log _{10} D
$$

où $S^{2}$ et $D$ sont respectivement la variance et la moyenne des effectifs (la densité de criquets) par lancer pour une parcelle donnée.

On peut calculer le nombre de lancers à réaliser, $n$, qui dépend donc de la densité à estimer, $D$, et de la précision recherchée, $p$, pour cet estimateur (Williams et al. 2002), en se fondant sur la définition de l'intervalle de confiance (CI) de la moyenne estimée :

$$
\text { Eq 2: } \mathrm{CI}=p=t_{1-\alpha / 2} S / \sqrt{n}
$$

où $S$ est la variance des effectifs, $t_{1-\alpha / 2}$ est le $t$ de Student.

Si on dispose d'une estimation de $S$ par modélisation de la loi de Taylor, on peut calculer le nombre $n$ de lancers à réaliser pour atteindre une précision $p$, ou calculer la précision $p$ en fonction du nombre de lancers $n$ :

$$
\begin{aligned}
& \text { Eq 3: } p=t_{1-\alpha / 2} \sqrt{a D^{b} / n} \\
& \text { Eq 4: } n=t_{1-\alpha / 2}^{2} a D^{b} / p^{2}
\end{aligned}
$$

Nous avons estimé par régression linéaire les paramètres de la relation de Taylor pour l'ensemble des criquets toutes espèces confondues, pour $C$. italicus et $P$. giornae. Les effets de l'année et de la surface de la parcelle échantillonnée sur la relation de Taylor ont été testés en les ajoutant à l'Eq 1 .

L'estimation des paramètres de cette relation a ensuite permis de calculer la précision associée à $n=10, n=15$ et $n=20$ lancers par parcelle en se fondant sur l'équation Eq 3. Ces valeurs de taille d'échantillon nous semblent compatibles avec une utilisation de la technique à grande échelle. Réciproquement, on a calculé le nombre de lancers nécessaires pour atteindre une précision recherchée (Eq 4). Les données ont été analysées avec le logiciel R, version 2.13.00 (R Development Core team 2009) en utilisant des modèles linéaires et la fonction AIC (Akaike 1974) qui permet de comparer des modèles entre eux.

\section{Résultats}

\section{Effets de la température sur l'estimation d'abondance des criquets}

Parmi les variables explicatives de la densité de criquets nous recherchionsun possible biais d'estimation lié à la température durant l'échantillonnage. La figure 2 présente la courbe de tendance et les valeurs observées de la densité estimée de criquets (toutes espèces confondues) en fonction de la température durant l'échantillonnage pour chaque année. On ne remarque visuellement aucune tendance, en particulier durant les années 2007, 2008 et 2009. Cette observation est confirmée par les résultats des modèles linéaires qui font apparaître que la température n'explique aucune part de la variabilité des densités estimées malgré des variations importantes de la température lors des échantillonnages (2005:F=0,50, $P=0,48, \mathrm{df}=1$, 92; $2006: F=0,94, P=0,33, \mathrm{df}=1,89 ; 2007: F=$ $0,001, P=0,95, \mathrm{df}=1,157 ; 2008: F=2,44, P=0,12$, $\mathrm{df}=1,214 ; 2009: F=0,22, P=0,64, \mathrm{df}=1,101$; $2010: F=0,004, P=0,94, \mathrm{df}=1,119)$. L'année 2007 est la plus froide $\left(23,5^{\circ} \mathrm{C}\right.$ en moyenne) tandis que l'année 2006 est la plus chaude $\left(27,7^{\circ} \mathrm{C}\right.$ en moyenne) (Tab. 1). Au sein de chaque année, on observe une gamme de températures durant l'échantillonnage assez large, la plus forte amplitude de $26^{\circ} \mathrm{C}$ étant observée en 2004 avec des températures variant de $10{ }^{\circ} \mathrm{C}$ à $36^{\circ} \mathrm{C}$. L'amplitude des températures est la plus faible durant les années fraîches $(2007,2008,2009)$ le minimum étant de $13{ }^{\circ} \mathrm{C}$ en 2007.

L'effet de la date d'échantillonnage n'est sensible qu'en 2005 avec une légère augmentation des effectifs 
Tableau 2. Paramètres estimés et résultats de la régression linéaire entre la variance (en log) et la densité (en log) de criquets $/ \mathrm{m}^{2}$ $\left(\log _{10} S^{2}=\log _{10} a+b \log _{10} m\right.$ ) pour toutes les espèces de criquets confondues (deux classes de densités), Pezotettix giornae et Calliptamus italicus (année 2007, autres années).

\begin{tabular}{|c|c|c|c|c|c|c|c|}
\hline \multicolumn{7}{|l|}{ Total criquets } & $\mathrm{df}$ \\
\hline $0,1-3 / m^{2}$ & 0,155 & 0,010 & 1,170 & 0,022 & 0,85 & 0,218 & 538 \\
\hline$>3 / m^{2}$ & 0,097 & 0,063 & 1,408 & 0,075 & 0,62 & 0,274 & 218 \\
\hline P. giornae & 0,267 & 0,014 & 1,300 & 0,018 & 0,93 & 0,175 & 383 \\
\hline \multicolumn{8}{|l|}{ C. italicus } \\
\hline 2007 & 0,356 & 0,046 & 1,414 & 0,061 & 0,93 & 0,165 & 40 \\
\hline Autres années & 0,124 & 0,016 & 1,142 & 0,022 & 0,93 & 0,143 & 206 \\
\hline
\end{tabular}

* $a=10^{\log 10 a-0.5 \operatorname{SE}(\log 10 a)}$ (Goldberger 1968)

durant la période d'échantillonnage (2005:F=2,92, $P=0,10, \mathrm{df}=1,92 ; 2006: F=0,23, P=0,63, \mathrm{df}=1$, 89; $2007: F=0,42, P=0,51, \mathrm{df}=1,157 ; 2008$ : $F=0,41, P=0,39, \mathrm{df}=1,214 ; 2009: F=1,14$, $P=0,29, \mathrm{df}=1,101 ; 2010: F=0,66, P=0,42$, $\mathrm{df}=1,119)$. Celui-ci s'est réalisé sur des périodes de temps relativement courtes (de 8 jours en 2005 jusqu'à 12 jours en 2009).

L'essentiel de la variabilité des densités est expliqué par le type de prairie (Poacées ou prairies artificielles de luzerne) (quelle que soit l'année : $F>15, P<0,001$ ). Les densités de criquets, toutes espèces confondues, sont plus élevées dans les prairies de graminées que dans les prairies de luzerne.

Tableau 3. Nombre de lancers à réaliser par parcelle pour estimer la densité de criquets toutes espèces confondues par $\mathrm{m}^{2}$ avec une précision absolue de $\pm 0,5 / \mathrm{m}^{2}, \pm 1 / \mathrm{m}^{2}, \pm 2 / \mathrm{m}^{2}$ (risque $\alpha=0,10$ ).

\begin{tabular}{c|c:c:c}
\hline Densité de criquets & \multicolumn{3}{|c}{ Nombre de lancers pour atteindre } \\
& $0,5 / \mathrm{m}^{2}$ & $1 / \mathrm{m}^{2}$ & $2 / \mathrm{m}^{2}$ \\
\hline $\mathbf{0 , 5}$ & 8 & - & - \\
$\mathbf{1 , 0}$ & 17 & - & - \\
$\mathbf{1 , 5}$ & 26 & 7 & - \\
$\mathbf{2 , 0}$ & 37 & 10 & - \\
$\mathbf{3 , 0}$ & 58 & 15 & - \\
$\mathbf{4 , 0}$ & 93 & 24 & 6 \\
$\mathbf{5 , 0}$ & $>100$ & 32 & 8 \\
$\mathbf{6 , 0}$ & $>100$ & 42 & 11 \\
$\mathbf{7 , 0}$ & $>100$ & 52 & 13 \\
$\mathbf{8 , 0}$ & $>100$ & 62 & 16 \\
$\mathbf{9 , 0}$ & $>100$ & 73 & 19 \\
$\mathbf{1 0 , 0}$ & $>100$ & 85 & 22 \\
$\mathbf{1 5 , 0}$ & $>100$ & $>100$ & 38 \\
$\mathbf{2 0 , 0}$ & $>100$ & $>100$ & 57 \\
$\mathbf{2 5 , 0}$ & $>100$ & $>100$ & 77 \\
\hline
\end{tabular}

\section{Modélisation de la variabilité des comptages et plan d'échantillonnage pour l'estimation d'abondance des criquets.}

Les densités de criquets toutes espèces confondues présentent des gammes de variation qui diffèrent selon les années. Ainsi, les plus fortes variations sont observées en 2006 avec des densités de 0 à 50,8 criquets $/ \mathrm{m}^{2}$ selon les parcelles tandis qu'en 2008 la densité la plus élevée était de 9,8 criquets $/ \mathrm{m}^{2}$. L'analyse de la relation qui lie la variance à la moyenne des densités montre que la variance augmente de manière exponentielle avec la densité. Une relation de Taylor unique ne peut cependant pas être établie sur l'ensemble des années, les paramètres $a$ et $b$ différant significativement de l'une à l'autre $(F=2,65 ; \mathrm{df}=746,758 ; P<0,01)$. Les données ont alors été séparées en deux groupes en fonction des densités $\left(\leq 3 / \mathrm{m}^{2}\right.$ ou $\left.>3 / \mathrm{m}^{2}\right)$ et ont été analysées séparément. Avec ce découpage, les paramètres $a$ et $b$ de la relation de Taylor ne diffèrent plus entre les années que ce soit pour les densités $\leq 3 / \mathrm{m}^{2}(F=1,51 ; \mathrm{df}=526,538 ; P=0,12)$ ou pour les densités $>3 / \mathrm{m}^{2}(F=1,54 ; \mathrm{df}=206,218 ; P=0,11)$. La surface de la parcelle qui varie de 0,04 ha à 12,24 ha n'intervient pas significativement dans les relations variance-moyenne de Taylor établies pour les deux classes de densités. Le tableau 2 présente les paramètres estimés des deux grandes classes de densités. A partir des paramètres de la classe $\leq 3 / \mathrm{m}^{2}$ nous avons calculé les tailles d'échantillons pour des densités variant de 0,1 à 3 criquets $/ \mathrm{m}^{2}$ et à partir des paramètres de la classe $>3 / \mathrm{m}^{2}$ celles pour des densités au-delà de 3 criquets $/ \mathrm{m}^{2}$.

Les tailles d'échantillon préconisées pour obtenir des précisions absolues ou relatives fixées à l'avance varient donc en fonction de la densité de criquets à estimer. Le nombre de lancers par parcelle augmente avec la densité de criquets à estimer pour une précision absolue donnée (tab. 3). Une précision absolue de $\pm 0,5$ criquets $/ \mathrm{m}^{2}$ 
impose des échantillons de plus de 50 lancers par parcelle dès lors que la densité est $>2,7$ criquets $/ \mathrm{m}^{2}$ (tab. 3). Lorsque la précision recherchée est de $\pm 1 / \mathrm{m}^{2}$, le nombre de lancers est considérablement diminué et reste inférieur à 50 lancers par parcelle pour des densités $<7,0$ criquets $/ \mathrm{m}^{2}$. Enfin, la précision de $\pm 2 / \mathrm{m}^{2}$ est atteinte avec moins de 30 lancers par parcelle pour les densités $<12,5$ criquets $/ \mathrm{m}^{2}$.

Dansla pratique, il apparait plusfacile dedéterminer une taille d'échantillon a priori que de l'adapter en fonction de la densité observée sur le terrain. Ainsi, nous avons calculé les précisions absolue (Fig. 3) et relative (Fig. 4) pour des tailles d'échantillons fixes de 10, 15 et 20 lancers par parcelle. Dix lancers par parcelle permettent d'obtenir une précision absolue qui varie de $\pm 0,16 / \mathrm{m}^{2}$ pour les densités les plus faibles à environ $\pm 6 / \mathrm{m}^{2}$ pour les densités $>28$ criquets $/ \mathrm{m}^{2}$. Cela correspond à des précisions relatives de 160 à $21 \%$. Si on considère les densités moyennes observées de 2004 à 2010, on remarque que ce nombre de lancers conduit en moyenne à des précisions relatives de $70 \%$ (en 2008) à 35\% (2004). Doubler le nombre de lancers permet d'améliorer la précision des estimateurs avec des précisions relatives qui varient de $50 \%$ pour les plus faibles densités à $15 \%$ pour les plus élevées.

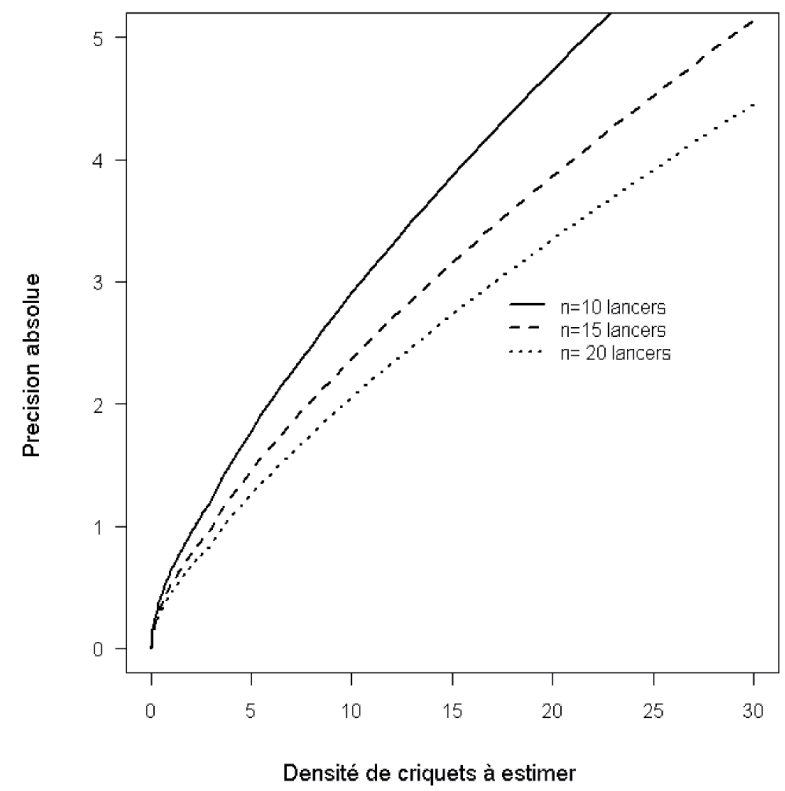

Figure 3

Précision absolue associée à l'estimation de la densité de criquets $/ \mathrm{m}^{2}$ pour des tailles d'échantillons de 10, 15 ou 20 lancers par parcelle en fonction de la densité de criquets à estimer (risque $\alpha=0,10$ ).
Les mêmes analyses que ci-dessus ont été réalisées pour les deux espèces, $P$. giornae et $C$. italicus. Ces deux espèces sont fréquentes dans la zone d'étude, mais leurs densités demeurent faibles (tab. 1) même si elles peuvent être élevées ponctuellement (la densité maximale enregistrée atteint 8,6 individus/ $\mathrm{m}^{2}$ pour $C$. italicus et 10,6 individus $/ \mathrm{m}^{2}$ pour $P$. giornae). Comme précédemment, on observe que la variance des comptages augmente avec la densité. La loi de Taylor modélise bien cette relation pour les deux espèces (tab. 2). Dans le cas de P. giornae on observe que l'effet de la surface de la parcelle sur cette relation est significatif. Toutefois, le paramètre associé à cette variable est très faible (paramètre $=$ $\left.1,35 \times 10^{-6}, \mathrm{SE}=6,5 \times 10^{-7}, P=0,05\right)$. Pour cette espèce, une relation commune pour toutes les années est trouvée $(F=0,70 ; \mathrm{df}=372,384 ; P=0,78)$. Ce n'est pas le cas de $C$. italicus, pour lequel l'année 2007 se démarque de l'ensemble des autres années qu'on peut caractériser par une relation unique (Tab. 2). La surface de la parcelle n'est pas une variable qui explique significativement les variations chez $C$. italicus. Nous ne développons pas de procédure d'échantillonnage de ces deux taxons dans cet article, quoique les paramètres estimés le permettent.

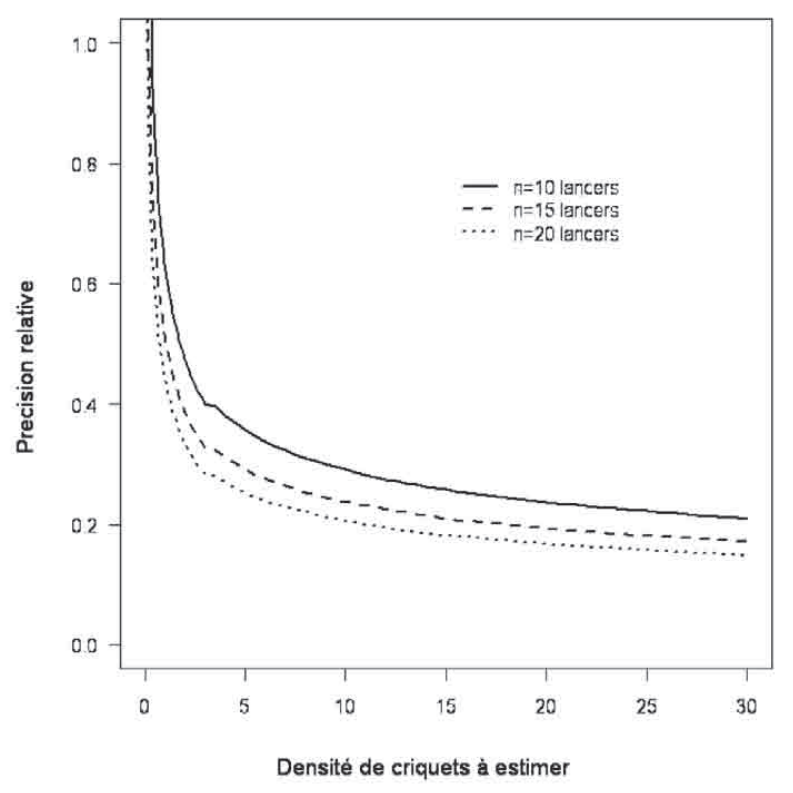

Figure 4

Précision relative associée à l'estimation de la densité de criquets $/ \mathrm{m}^{2}$ pour des tailles d'échantillons de 10, 15 ou 20 lancers par parcelle en fonction de la densité de criquets à estimer (risque $\alpha=0,10$ ). 


\section{Discussion}

\section{Fiabilité de la technique d'échantillonnage vis-à-vis des conditions d'échantillonnage}

L'échantillonnage quantitatif des populations de criquets rencontre certaines difficultés liées essentiellement à deux caractéristiques de leur biologie : d'une part ils fuient en sautant et en volant à l'approche de l'observateur, et d'autre part leur activité est fortement liée aux conditions météorologiques, en particulier la température, l'éclairement, la pluviosité et le vent (Onsager 1977). De manière générale, les conditions d'échantillonnage sont mentionnées comme étant des facteurs limitants pour la comparaison de données de dénombrement de criquets issues de campagnes différentes (Gardiner et al. 2005). La tendance des biais est la sous-estimation des effectifs (Harvey \& Gardiner 2006). Dans notre étude, nous avons évalué l'effet de la température durant l'échantillonnage sur la densité estimée de criquets en utilisant la technique du biocénomètre d' $1 \mathrm{~m}^{2}$. Nos résultats indiquent que l'échantillonnage réalisé dans une gamme de température allant de $10{ }^{\circ} \mathrm{C}$ à $36^{\circ} \mathrm{C}$ n'induit pas de biais dans l'estimation des densités de criquets, à condition toutefois d'ajuster le temps de recherche des criquets dans l'enceinte en fonction de la température. Ainsi, par temps froid, on passe plus de temps à cette opération du fait de l'inactivité des criquets, tandis que par temps très chaud, on passe plus de temps à empêcher les criquets de sortir de l'enceinte et à les recapturer une fois échappés du fait de leur activité plus grande. L'absence d'ajustement du temps passé peut être à l'origine d'une sous-estimation des effectifs aux températures extrêmes, froides ou chaudes. Ces résultats confirment ceux que nous avions obtenus précédemment avec une autre méthodologie (Badenhausser et al. 2009) sur un jeu limité de parcelles.

Dans notre étude, les relevés ont été réalisés sur des périodes de temps relativement limitées, soit entre 8 et 12 jours, pour minimiser les variations de densité entre parcelles dues à la date d'échantillonnage. Ces variations peuvent en effet être importantes (Badenhausser et al. 2009) du fait des processus de mortalité et de dispersion. Ainsi, en 2006 on a pu observer dans certaines parcelles des baisses de $25 \%$ des effectifs d'adultes de $C$. italicus entre le 7 et le 15 août dues à une forte dispersion (Badenhausser et al. 2009). La durée de la période d'échantillonnage sur laquelle nous avons basé notre étude n'a pas induit de biais dans les estimations de densité, même si en 2005 ce facteur était significatif à 10\%. Il convient donc, selon l'objectif des suivis, de minimiser la durée de la période d'échantillonnage ou de procéder à un calibrage des effectifs selon la date d'échantillonnage. Cette dernière solution implique de disposer d'un suivi temporel de la dynamique des populations locales sur plusieurs parcelles de référence.

On peut souligner que les résultats observés dans notre étude s'appliquent à des communautés de criquets composées à plus de $90 \%$ d'espèces de la sous-famille des Gomphocerinae ainsi que du Catantopinae $P$. giornae. Ces espèces ont des capacités de déplacement instantanées qui demeurent limitées dans l'espace (Reinhardt et al. 2005 ; Picaud \& Petit 2007). Ainsi, l'échantillonnage ciblé d'autres espèces beaucoup plus mobiles comme $A$. thalassinus, $O$. caerulescens ou à un degré moindre $C$. italicus, peut s'avérer difficile voire impossible avec la technique du biocénomètre. Pour ces espèces, la simple perturbation liée au déplacement de l'observateur suffit à provoquer l'envol des individus, en particulier par temps chaud et quand les densités de criquets sont élevées.

\section{Procédure d'échantillonnage}

Comme de nombreux autres groupes d'invertébrés, les criquets présentent de fortes variations de densité à la fois dans l'espace et dans le temps (Badenhausser et al. 2009 ; Richard \& Waloff 1954). Les variations locales d'abondance (intra-parcelles) sont connues chez les criquets et traduisent des variations locales de l'habitat qui sont plus ou moins favorables aux différentes espèces (Guido \& Gianelle 2001). Les variations entre parcelles et entre années sont liées à des variables climatiques (Kohler et al. 1999) qui agissent à différentes échelles, au contexte paysager (Marini et al. 2010) mais aussi à de nombreux autres facteurs comme la prédation, la disponibilité et la qualité de la ressource qui sont fortement influencées par les pratiques de gestion et d'exploitation (Guido \& Gianelle 2001; Kruess \& Tscharntke 2002 ; Wingerden et al. 1992).

Conformément à la loi de Taylor, nous observons que la variance des densités de criquets $/ \mathrm{m}^{2}$ augmente de manière exponentielle avec leur moyenne qu'il s'agisse de toutes les espèces confondues, de $P$. giornae ou de $C$. italicus. Toutes espèces confondues, ce sont les Gomphocerinae, en particulier E. elegantulus et $C$. biguttulus, qui représentent l'essentiel des densités de criquets (tab. 1). Pour ce groupe, nous observons quà des densités $>3$ criquets $/ \mathrm{m}^{2}$, et le paramètre $b$ de la loi de Taylor est plus élevé $(b=1,408)$ que pour les densités $<3$ criquets $/ \mathrm{m}^{2}(b=1,170)$. Ce paramètre est interprété comme un indice d'agrégation (Taylor 1961) ce qui traduit une répartition plus agrégative des individus aux fortes densités (Taylor 1984). Ces paramètres sont très proches de ceux que nous avions établis dans une étude précédente où les années étaient peu contrastées au 
regard des densités de criquets (densités $\leq 3: b=1,193$; densités $>3: b=1,559)$ (Badenhausser et al. 2007). Legg \& Lockwood (1995) ont mentionné des seuils de densités comparables pour définir les techniques d'échantillonnage les plus appropriées. Il s'ensuit que les procédures d'échantillonnage doivent être adaptées en fonction de la densité observée sur le terrain. Ainsi, le nombre de lancers à réaliser pour estimer la densité de criquets $/ \mathrm{m}^{2}$ avec une précision fixée est fonction de la densité et augmente avec elle. Pratiquement, il est plus simple pour planifier les opérations de terrain de fixer un nombre de lancers à l'avance plutôt que de le définir au fur et à mesure de l'échantillonnage de la parcelle. De plus, ce nombre est contraint par la faisabilité de la procédure notamment par son coût. Les observations que nous réalisons nécessitent la plupart du temps d'avoir un grand nombre de parcelles échantillonnées sur une période de temps qui n’induit pas de biais significatif sur les estimations de densité des populations. Nous avons choisi selon les objectifs des échantillonnages, des tailles d'échantillons de 10 à 15 lancers par parcelle. Le temps d'échantillonnage d'une parcelle est directement lié à la densité des criquets qui s'y trouvent (Badenhausser et al. 2007). Ce nombre de lancers permet d'échantillonner une parcelle en 20 à 30 minutes dès lors que la densité est $<3$ criquets $/ \mathrm{m}^{2}$ et qu'on ne prélève pas les individus. Avec une telle taille d'échantillon, les précisions qu'on peut atteindre telles que nous les avons définies (densités estimées $\pm \mathrm{CI}$ ) varient de $50 \%$ pour les densités les plus faibles $\left(<1 / \mathrm{m}^{2}\right)$ à $30 \%$ pour les densités intermédiaires $\left(2 / \mathrm{m}^{2}\right.$ $<$ densités $\left.<7 / \mathrm{m}^{2}\right)$ et à $20 \%$ au delà. Ces niveaux de précision sont à rapprocher des objectifs des études mises en œuvre et des différences qu'on souhaite mettre en évidence.

Dans les milieux agricoles intensifs que nous avons échantillonnés (majoritairement des prairies temporaires de Poacées ou de légumineuses), la surface de la parcelle n'a pas d'effet sur $C$. italicus ni sur les criquets toutes espèces confondues, ce qui confirme ou suggère que ces espèces sont colonisatrices. Inversement, la surface de la parcelle a un impact sur $P$. giornae, espèce non colonisatrice. Dans ces paysages, les habitats sont fragmentés et les prairies peu nombreuses. On ne peut pas exclure que dans d'autres contextes moins intensifs, la variabilité des effectifs de criquets $/ \mathrm{m}^{2}$ augmente avec la surface des milieux peut-être plus hétérogènes.

\section{Conclusion}

La technique et la procédure d'échantillonnage proposées dans cet article permettent d'estimer l'abondance de populations de criquets, appartenant essentiellement à des espèces de Gomphocerinae dans des gammes de densités modérées et dans les milieux prairiaux associés aux paysages agricoles intensifs. Lintérêt de la technique du biocénomètre réside dans sa capacité à fournir des estimations par unité de surface qui peuvent donc servir de base pour estimer des effectifs à grande échelle. Cette technique semble assez robuste vis à vis des conditions d'échantillonnage et des structures de végétation rencontrées dans les écosystèmes prairiaux de ce contexte paysager (cultures de luzerne, prairies de graminées ou d'associations exploitées). Sa mise en œuvre au travers de procédures d'échantillonnage aléatoires est compatible avec la pratique en termes de précision associée aux estimateurs et de faisabilité économique.

Remerciements. La conception du modèle de biocéomètre démontable a bénéficié successivement de la collaboration technique de J.P. Frankowski, L. Bruneteau, M. Vandier, P. Simon et D. Simon. Nous remercions les personnes qui ont réalisé le travail de terrain en espérant ne pas en oublier: J. Amouret, P. Amouroux, E. Baron, F. Bouchon, L. Bruneteau, A. Desternes, N. Edouard, N. Guillon, B. Hippert, S. Lorioux, A. Morel, N. Paris Le Clerc, L. Pommarel, O. Rollin, M. Roncoroni, M. Vandier, S. Vandier, ainsi que l'équipe technique qui a réalisé les biocénomètres : L. Bruneteau, N. Guillon, T. Breda, D. Simon, P. Simon, M. Vandier.

\section{Références}

Akaike H. 1974. A new look at the statistical model identification. IEEE Transactions on Automatic Control 19: 716-723.

Badenhausser I., Amouroux P., Bretagnolle V. 2007. Estimating acridid (Orthoptera: Acrididae) densities in grassland habitats: A comparison between presence-absence and abundance sampling designs. Environmental Entomology 36: 1494-1503.

Badenhausser I., Amouroux P., Lerin J., Bretagnolle V. 2009. Acridid (Orthoptera: Acrididae) abundance in Western European Grasslands: sampling methodology and temporal fluctuations. Journal of Applied Entomology 133: 720-732.

Baldi A., Kisbenedek T. 1997. Orthopteran assemblages as indicators of grassland naturalness in Hungary. Agriculture, Ecosystems \& Environment 66:121-129.

Barker A. M. 2004. Insect as food for farmland birds - is there a problem? p. 37-50 in: Van Emden H., Rothschild M. (eds.) Insect and bird interactions. Intercept Ltd, Hampshire, UK.

Bretagnolle V., Villers A., Denonfoux L., Badenhausser I. 2011. Rapid recovery of a depleted population of Little Bustards Tetrax tetrax following provision of alfalfa through an agri-environment scheme. Ibis 153: 4-13.

Browde J., Pedigo L., Degooyer T., Highley L., Wintersteen W., Zeiss M. 1992. Comparison of sampling techniques for grasshoppers (Orthoptera: Acrididae) in soybean. Journal of Economic Entomology 85: 2270-2274.

Dangerfield J.M., Pik A.J., Britton D., Holmes A., Gillings M., Oliver I., Briscoe D., Beattie A. J. 2003. Patterns of invertebrate biodiversity across a natural edge. Austral Ecology 28: 227-236.

Doxon E.D., Davis C.A., Fuhlendorf S. D. 2011. Comparison of two methods for sampling invertebrates: vacuum and sweep-net sampling. Journal of Field Ornithology 81: 60-67.

ESRI 2006. ESRI ArcMAP 9.3. www.esri.com. 
Evans E., Rogers R., Opfermann D. 1983. Sampling grasshoppers (Orthoptera: Acrididae) on burned and unburned tallgrass prairie: night trapping vs. sweeping. Environmental Entomology 12:1449-1454.

Gardiner T., Pye M., Field R. Hill J. 2002. The influence of sward height and vegetation composition in determining the habitat preferences of three Chorthippus species (Orthoptera: Acrididae) in Chelmsfors, Essex, UK. Journal of Orthoptera Research 11: 207-213.

Gardiner T., Hill J., Chesmore D. 2005. Review of the methods frequently used to estimate the abundance of Orthoptera in grassland ecosystems. Journal of Insect Conservation 9: 151-173.

Green R.E., Balmford A., Crane P.R., Mace G.M., Reynolds J. D., Turner R.K. 2005. A framework for improved monitoring of biodiversity: Responses to the World Summit on Sustainable Development. Conservation Biology 19: 56-65.

Gueguen A., Lefeuvre J. C., Forgeard F., Touffet J. 1980. Analyse comparée de la dynamique de la restauration du peuplement d'orthoptères et du peuplement végétal dans une zone brûlée de lande. Bulletin Ecologie 11: 747-764.

Guido M., Gianelle D. 2001. Distribution patterns of four Orthoptera species in relation to microhabitat heterogeneity in an ecotonal area. Acta Oecologica 22:175-185.

Harvey P., Gardiner T. 2006. Pitfall trapping of scarce Orthoptera at a coastal nature reserve in Essex, UK. Journal of Insect conservation 10: 371-373.

Inchausti P., Bretagnolle V. 2005. Predicting short-term extinction risk for the declining Little Bustard (Tetrax tetrax) in intensive agricultural habitats. Biological Conservation 122: 375-384

Kohler G., Perner J., Schumacher J. 1999. Grasshopper population dynamics and meteorological parameters - lessons from a case study. Ecography 22: 205-212.

Kruess A., Tscharntke T. 2002. Grazing intensity and the diversity of grasshoppers, butterflies, and trap-nesting bees and wasps. Conservation Biology 16: 1570-1580.
Legg D., Lockwood J. 1995. Estimating densities of grasshoppers (Orthoptera: Acrididae) assemblages using binomial sampling technique. Journal of the Kansas Entomological Society 68: 178-183.

Marini L., Bonmarco R., Fontana P., Battisti A., 2010. Disentangling effects of habitat diversity and area on orthopteran species with contrasting mobility. Biological Conservation 143: 2164-2171.

Onsager J. 1977. Comparison of five methods for estimating density of rangeland grasshoppers. Journal of Economic Entomology 70:187-190.

Picaud F., Petit D. P. 2007. Primary succession of Acrididae (Orthoptera): Differences in displacement capacities in early and late colonizers of new habitats. Acta Oecologica-International Journal of Ecology 32: 59-66.

R Development Core Team 2011. R: A Language and Environment for Statistical Computing. Foundation for Statistical Computing, Vienna, Austria. ISBN 3-900051-07-0, URL http://www.R-project.org/.

Reinhardt K., Kohler G., Maas S., Detzel P. 2005. Low dispersal ability and habitat specificity promote extinctions in rare but not in widespread species: the Orthoptera of Germany. Ecography 28:593-602.

Richards O.W., Waloff M.A., Waloff N. 1954. Studies on the biology and population dynamics of british grasshoppers. Anti-Locust bulletin 17.

Schirmel J., Buchholz S., Fartmann T. 2010. Is pitfall trapping a valuable sampling method for grassland Orthoptera? Journal of Insect Conservation 14: 289-296.

Taylor L.R. 1961. Aggregation, variance and the mean. Nature (Lond.) 4: $732-735$.

Taylor L.R. 1984. Assessing and interpreting the spatial distributions of insect populations. Annual Review of Entomology 29: 321-357.

Voisin J.-F. 1986. Une méthode simple pour caractériser l'abondance des orthoptères en milieu ouvert. L'Entomologiste 42:113-119.

Williams B.K., Nichols J.D., Conroy M.J. 2002. Analysis and management of animal populations. Academic Press, New-York.

Wingerden W., Kreveld A., Bongers W. 1992. Analysis of species composition and abundance of grasshoppers (Orth., Acrididae) in natural and fertilized grasslands. Journal of Applied Entomology 113: 138-152. 